ESAIM: PROCEEDINGS, April 2012, Vol. 36, p. 170-179

Danièle Fournier-Prunaret, Laura Gardini, \& Ludwig Reich, Editors

\title{
COEXISTING CYCLES IN A CLASS OF 3-D DISCRETE MAPS
}

\author{
ANNA AGLIARI ${ }^{1}$
}

\begin{abstract}
In this paper we consider the class of three-dimensional discrete maps $M(x, y, z)=$ $[\phi(y), \phi(z), \phi(x)]$, where $\phi: \mathbb{R} \rightarrow \mathbb{R}$ is an endomorphism. We show that all the cycles of the 3-D map $M$ can be obtained by those of $\phi(x)$, as well as their local bifurcations. In particular we obtain that any local bifurcation is of co-dimension 3, that is three eigenvalues cross simultaneously the unit circle. As the map $M$ exhibits coexistence of cycles when $\phi(x)$ has a cycle of period $n \geq 2$, making use of the Myrberg map as endomorphism, we describe the structure of the basins of attraction of the attractors of $M$ and we study the effect of the flip bifurcation of a fixed point.
\end{abstract}

AMS (2000) subject classification. 37B99, 37G35, 37M05.

Keywords. 3-D discrete maps, Periodic orbits, Bifurcations of co-dimension 3.

Résumé. Dans ce papier nous considérons la classe des applications trois-dimensionnelles discrètes $M(x, y, z)=[\phi(y), \phi(z), \phi(x)]$, où $\phi: \mathbb{R} \rightarrow \mathbb{R}$ est un endomorphisme. Nous montrons que tous les cycles de l'application 3-D $M$ peuvent etre obtenus par ceux de $\phi(x)$, ainsi que leurs bifurcations locales. En particulier, nous obtenons que toute bifurcation locale est de co-dimension 3, c'est-à-dire que trois valeurs propres franchissent simultanément le cercle unité. Comme l'application $M$ exhibe une coexistence de cycles lorsque $\phi(x)$ a un cycle de période $n \geq 2$, en utilisant l'application de Myrberg comme endomorphisme, nous décrivons la structure des bassins d'attraction des attracteurs de $M$ et nous étudions les effets d'une bifurcation de doublement de période d'un point fixe.

Mots clefs. Transformation à temps discret de dimension 3, Orbites périodiques, Bifurcations de codimension 3 .

\section{INTRODUCTION}

In 2000 Bischi, Mammana and Gardini, [2], have studied the class of two-dimensional discrete maps of form $N(x, y)=\left[r_{1}(y), r_{2}(x)\right]$, having the property that their second iterate is a decoupled map. They proved that cycles and more complex attractors of such a map can be obtained by the study of the one-dimensional map $F(x)=r_{1}\left(r_{2}(x)\right)$ as well as the bifurcations they undergo. Moreover in [2], it is also proved that, in general, maps like $N$ are characterized by multistability, i.e., coexistence of many different attractors, that may be stable periodic cycles or cyclical chaotic attractors. Coexisting cycles of $N$ exist not only when the map $F$ has two or more coexisting cycles but also when $F$ has a unique cycle of period $n>2$.

More recently Agliari, Fournier-Prunaret and Taha, [1], have extended the results of [2] to the threedimensional maps having the property that their third iterate has separate components, that is to $T(x, y, z)=$ $[f(y), g(z), h(x)]$. As in the case of 2D-maps, in [1] it is shown that the attractors of $T$ may be derived from those of $H(x)=f(g(h(x)))$ as well as their stability properties. The 3D-phase plane only makes more complex

${ }^{1}$ Dept. Economic and Social Science, Catholic University, via Emilia Parmense 84, 49100 Piacenza, Italy; email: anna.agliari@unicatt.it.

(c) EDP Sciences, SMAI 2012 
the classification of the cycles and increases the number of the coexisting cycles of $T$. In particular, in [1] it is also shown that if $H$ has a unique cycle of period $n>1$ then $T$ exhibits coexistence of cycles.

In the present paper we consider a particular case of maps having separate third iterate, obtained by assuming that $f=g=h$. Our aim is to give a fairly general description of the cycles of $M(x, y, z)=[\phi(y), \phi(z), \phi(x)]$ and, by means of an example, to explain the structure of the basins of attraction of the stable ones.

We show that in such a particular case all the cycles of the 3D map $M$ can be obtained starting simply from those of $\phi(x)$. We classify such cycles as homogeneous, if they are obtained by a unique cycle of $\phi(x)$, mixed cycles, if they are obtained by the combination of the periodic points of two coexistent cycles of $\phi(x)$, and pure mixed cycles, if their periodic points are obtained combining three coexisting cycles of $\phi(x)$. The eigenvalues of the cycles of $M$, as well, can be obtained by the multipliers of the associated cycles of $\phi(x)$ and any local bifurcation occurring for the one-dimensional map is reflected into a local bifurcation of the map $M$ associated with three eigenvalues crossing the unit circle simultaneously (i.e., they are codimension 3 bifurcations).

As the map $M$ exhibits coexistence of cycles when $\phi(x)$ has a cycle of period $n \geq 2$, making use of the Myrberg map as endomorphism, we also describe the structure of the basins of attraction of the attractors of $M$ and we study the effect of the flip bifurcation of a fixed point.

Our results are analogous to, and extend, those obtained in the 2D case by Tramontana, Gardini and Puu in [4].

\section{THE CLASS OF 3-D MAPS}

We consider the class of three-dimensional maps defined by

$$
M\left(x_{t}, y_{t}, z_{t}\right):\left\{\begin{array}{l}
x_{t+1}=\phi\left(y_{t}\right) \\
y_{t+1}=\phi\left(z_{t}\right) \\
z_{t+1}=\phi\left(x_{t}\right)
\end{array}\right.
$$

where $\phi: \mathbb{R} \rightarrow \mathbb{R}$ is an endomorphism and $t \in \mathbb{Z}$.

The interesting property of $M$ in (1) is that its third iterate has separate components, being $M^{3}(x, y, z)=$ $\left[\phi^{3}(x), \phi^{3}(y), \phi^{3}(z)\right]$. Such a property implies, as we shall see, that the invariant sets of $M$ may be deduced by the analysis of the one-dimensional map $\phi(x)$.

The class of map we are considering is a particular case of

$$
T\left(x_{t}, y_{t}, z_{t}\right):\left\{\begin{array}{l}
x_{t+1}=f\left(y_{t}\right) \\
y_{t+1}=g\left(z_{t}\right) \\
z_{t+1}=h\left(x_{t}\right)
\end{array}\right.
$$

whose third iterate has separate components, as well. The map $T$ in (2) has been recently studied by Agliari, Fournier-Prunaret and Taha, [1], and, for the convenience of the reader, we briefly resume the main results they have obtained.

Let us consider the one-dimensional map $H(x)=f(g(h(x)))$, the first component of $T^{3}$ from which the invariant sets of the map $T$ can be obtained. Indeed, $\left\{x_{1}, x_{2}, \ldots, x_{n}\right\}$ is a period $n$ cycle of $H$ if and only if $\left(x_{i}, g\left(h\left(x_{j}\right)\right), h\left(x_{l}\right)\right), i, j, l=1, \ldots, n$, is a periodic point of $T$, and consequently $\mathbf{P}=\left\{x_{1}, x_{2}, \ldots, x_{n}\right\} \times$ $\left\{g\left(h\left(x_{1}\right)\right), g\left(h\left(x_{2}\right)\right), \ldots, g\left(h\left(x_{n}\right)\right)\right\} \times\left\{h\left(x_{1}\right), h\left(x_{2}\right), \ldots, h\left(x_{n}\right)\right\}$ is the set of all the periodic points of $T$.

In addiction, we are able to classify all cycles of the map $T$ based on the cycles of $H$, as stated in the following claims.

We start considering a single cycle of $H$ and the associated cycles of $T$, called homogeneous cycles.

Claim. (Homogeneous cycles) Let $\left\{x_{1}, x_{2}, \ldots, x_{n}\right\}$ be a cycle of prime period $n$ of $H$ with multiplier $\lambda$.

- If $n$ is not a multiple of 3 then $T$ has 
-1 cycle of period $n$, with eigenvalues $\lambda_{1}=\sqrt[3]{\lambda}, \lambda_{2}=\sqrt[3]{\lambda} \frac{-1+i \sqrt{3}}{2}, \lambda_{3}=-\sqrt[3]{\lambda} \frac{1+i \sqrt{3}}{2}$

$-\frac{n^{2}-1}{3}$ cycles of period $3 n$, with eigenvalues $\lambda_{1}=\lambda_{2}=\lambda_{3}=\lambda$;

- if $n$ is a multiple of 3 then $T$ has

$-\frac{n^{2}}{3}$ cycles of period $3 n$, with eigenvalues $\lambda_{1}=\lambda_{2}=\lambda_{3}=\lambda$.

Note that if $n>1$, the map $T$ exhibits coexistence of cycles.

Obviously, also the coexisting cycles of $H$ generate coexisting cycles of $T$. Let us assume that the map $H$ has three coexisting cycles $A=\left\{x^{A}\right\}, B=\left\{x^{B}\right\}$ and $C=\left\{x^{C}\right\}$ of prime period $n, m$ and $p$, respectively, and let $\lambda_{i}, i=A, B, C$ be the corresponding multipliers. In such a case the set $\mathbf{P}$ of the periodic points of $T$ contains: (i) the triplets $\left(x_{i}^{s}, g\left(h\left(x_{j}^{s}\right)\right), h\left(x_{l}^{s}\right)\right)$ the components of which all belong to the same cycle $s(s=A, B, C)$ and then to some homogeneous cycle; (ii) the triplets $\left(x_{i}^{s}, g\left(h\left(x_{j}^{t}\right)\right), h\left(x_{l}^{r}\right)\right)$ with $s, t, r=A, B, C$ and $s \neq t \neq r$, the components of which simultaneously belong to the three different cycles; (iii) the remaining triplets that have two components belonging to the same cycle and the third one to a different cycle. In the following we shall call mixed cycles as described in (iii) and pure mixed cycles as described in (ii).

Claim. (Mixed cycles) Any pair of coexisting cycles of $H$ of prime period $n$ and $m$ generate $(n+m) \frac{n m}{s}$ mixed cycles of $T$ of period $3 s$, where $s$ is the least common multiple $(L C M)$ of $m$ and $n$.

Moreover, if we consider, as an example, the two cycles $A$ and $B$ then the eigenvalues of the mixed cycles are either $\left(\lambda_{A}^{k_{1}}, \lambda_{A}^{k_{1}}, \lambda_{B}^{k_{2}}\right)$ or $\left(\lambda_{A}^{k_{1}}, \lambda_{B}^{k_{2}}, \lambda_{B}^{k_{2}}\right)$, where $k_{1}=s / n$ and $k_{2}=s / m$, depending on the number of periodic points of the two different cycles of $H$ that belong to them.

Claim. (Pure mixed cycles) If the map $H$ has three coexisting cycles of prime period $n, m$ and $p$ then the map $T$ has $2 \frac{n m p}{S}$ pure mixed cycles of period $3 S$, being $S=L C M(n, m, p)$. The eigenvalues of such cycles are $\left(\lambda_{A}^{k_{1}}, \lambda_{B}^{k_{2}}, \lambda_{C}^{k_{3}}\right)$, where $k_{1}=S / n, k_{2}=S / m$ and $k_{3}=S / p$.

As a final observation on the map $T$ in (2), we remark that a straight line parallel to the $z$-axis is mapped into a straight line parallel to the $y$-axis, a straight line parallel to the $y$-axis is mapped into a straight line parallel to the $x$-axis and a straight line parallel to the $x$-axis is mapped into a straight line parallel to the $z$-axis. Analogously, a plane parallel to the $x y$-plane is mapped into a plane parallel to the $x z$-plane, a plane parallel to the $x z$-plane is mapped into a plane parallel to the $y z$-plane, a plane parallel to the $y z$-plane is mapped into a plane parallel to the $x y$-plane. Such properties are important in order to study the stable and unstable sets of the saddle cycles of the map $T$. Indeed, a saddle cycles $S$ of $T$ is either a mixed or a pure mixed cycles of period a multiple of 3 and then the Jacobian matrix evaluated at $S$ is diagonal with eigenvectors parallel to the canonical basis $\left(e_{1}, e_{2}, e_{3}\right)$. Hence the local stable and unstable manifolds of $S$ belong either to planes or to straight lines parallel to some coordinate axis or plane.

Let us now come back to the map $M$ defined in (1), in order to apply the properties recalled above.

Before that, we remark that the invariant sets of the map $M$ have peculiar properties. Indeed, denoting by $\Delta$ the straight line $x=y=z$, we can formulate the two following propositions with trivial proofs.

Proposition 1. For the map $M$ in (1)

- $\Delta$ is a trapping set, i.e. $M(\Delta) \subseteq \Delta$;

- the restriction on $\Delta$ of the map $M$ is the one-dimensional map $\phi(x)$.

Proposition 2. Let us consider the isometries $S_{1}(x, y, z)=(y, z, x)$ and $S_{2}(x, y, z)=(z, x, y)$, then

- $M\left(S_{1}(x, y, z)\right)=S_{1}(M(x, y, z))$

- $M\left(S_{2}(x, y, z)\right)=S_{2}(M(x, y, z))$

From Proposition 2 we obtain that if $I$ is an invariant set of $M$ (i.e., $M(I)=I$ ), then it follows that either $S_{i}(I)=I$, and $I$ is included in $\Delta$, or $I^{\prime}=S_{i}(I)$ is an invariant set of $M$, being $S_{i}(I)=S_{i}(M(I))=M\left(S_{i}(I)\right)$, with $i=1,2$. 
Furthermore, by means of the isometries $S_{1}$ and $S_{2}$ the forward iterates of the map $M$ in (1) can be written in a easy way; indeed, denoting by $S_{0}$ the identity transformation, we have that for any $k \geq 1$

$$
M^{k}(x, y, z)=S_{r}\left(\phi^{k}(x), \phi^{k}(y), \phi^{k}(z)\right)
$$

where $r \equiv k(\bmod 3)$.

\section{The COEXISTing CYCLES OF $M$}

In this section we classify the cycles of the map $M$ defined in (1) starting from those of the map $\phi$. Indeed, as observed in the previous section, the third iterate of the map $M$ has separated components, as the map $T$ in (2), and then its cycles can be obtained by those of $\phi^{3}(x)$ (i.e., the first component of $M^{3}$ ) that, in turn, only depend on $\phi(x)$.

We start with the homogeneous cycles, proving the following

Proposition 3. Let $X=\left\{x_{i}\right\}_{i=1, \ldots, n}$ be a cycle of $\phi(x)$ of period $n \geq 1$, then

- $M$ has 1 cycle of period $n$ and $\frac{n^{2}-1}{3}$ cycles of period $3 n$, if $n$ is not a multiple of 3 ;

- $M$ has $n^{2}$ cycles of period $n$, if $n$ is a multiple of 9 ;

- $M$ has 3 cycles of period $\frac{n}{3}$ and $n^{2}-1$ cycles of period $n$, if $n$ is a multiple of 3 but $\frac{n}{3}$ isn't.

Proof. To prove the statement we make use of the claims on the cycles of $T$, looking at the cycles of $\phi^{3}(x)$.

If $n$ is not a multiple of 3 , then $X$ is a period $n$ cycle of $\phi^{3}(x)$ as well. Consequently $M$ has 1 cycle of period $n$ and $\frac{n^{2}-1}{3}$ cycle of period $3 n$. Moreover, due to Proposition 2, the cycle of period $n$ is an invariant set of the isometries $S_{1}$ and $S_{2}$, since it is the unique cycle of such a period. Then its periodic points are $\left(x_{i}, x_{i}, x_{i}\right)$, $i=1, \ldots, n$.

If $\mathrm{n}$ is a multiple of 9 , then $\phi^{3}(x)$ has 3 different cycles of period $\frac{n}{3}$. Being $\frac{n}{3}$ a multiple of 3 , each one of these cycles gives rise to $\frac{n^{2}}{9}$ cycles of period $3 \frac{n}{3}=n$. The leftover periodic points of the set $\left\{x_{1}, x_{2}, \ldots, x_{n}\right\}^{3}$ belong to cycles generated by the coexistence of the three cycles of $\phi^{3}(x)$ and are of period $n$, being $\frac{n}{3}$ the least common multiple among the periods. Then, in such a case, all the cycles of $M$ are of period $n$ and are $\frac{n^{3}}{n}=n^{2}$ in number.

If $\mathrm{n}$ is a multiple of 3 , but $\frac{n}{3}$ isn't, then $\phi^{3}(x)$ has 3 different cycles of period $\frac{n}{3}$. Being $\frac{n}{3}$ not a multiple of 3 , each one of these cycles gives rise to one cycle of period $\frac{n}{3}$ and $\frac{\frac{n^{2}}{9}-1}{3}=\frac{n^{2}-9}{27}$ cycles of period $n$. The leftover periodic points of the set $\left\{x_{1}, x_{2}, \ldots, x_{n}\right\}^{3}$ belong to cycles generated by the coexistence of the three cycles of $\phi^{3}(x)$ and are of period $n$, being $\frac{n}{3}$ the least common multiple among the periods. Summing up, in such a case, $M$ has 3 cycles of period $\frac{n}{3}$ and $\frac{n^{3}-n}{n}=n^{2}-1$ cycles of period $n$.

The cycles of period $\frac{n}{3}$ of $M$ existing when $n$ is a multiple of 3 but $\frac{n}{3}$ isn't can be easily identified. Indeed if we look for the fixed points of the map $M^{\frac{n}{3}}$, from (3) we obtain that

- if $\frac{n}{3} \equiv 1(\bmod 3)$ then the periodic points $\left(x_{i}, x_{i+2} \frac{n}{3}, x_{i+\frac{n}{3}}\right), i=1, \ldots, \frac{n}{3}$, belong to the same cycle

- if $\frac{n}{3} \equiv 2(\bmod 3)$ then the periodic points $\left(x_{i}, x_{i+\frac{n}{3}}, x_{i+2} \frac{n}{3}\right), i=1, \ldots, \frac{n}{3}$, belong to the same cycle.

The periodic points of these cycles do not belong to the invariant set $\Delta$, then, as implied by Proposition 2 , the three cycles of period $\frac{n}{3}$ have to be obtained each other by means of the isometries $S_{1}$ and $S_{2}$. Thus we have that

- if $\frac{n}{3} \equiv 1(\bmod 3)$ then the 3 different cycles of period $\frac{n}{3}$ can be found by starting from the periodic points $\left(x_{1}, x_{1+2 \frac{n}{3}}, x_{1+\frac{n}{3}}\right),\left(x_{1+2 \frac{n}{3}}, x_{1+\frac{n}{3}}, x_{1}\right),\left(x_{1+\frac{n}{3}}, x_{1}, x_{1+2 \frac{n}{3}}\right)$;

- if $\frac{n}{3} \equiv 2(\bmod 3)$ then 3 different cycles of period $\frac{n}{3}$ can be found by starting from the periodic points $\left(x_{1}, x_{1+\frac{n}{3}}, x_{1+2 \frac{n}{3}}\right),\left(x_{1+\frac{n}{3}}, x_{1+2 \frac{n}{3}}, x_{1}\right),\left(x_{1+2 \frac{n}{3}}, x_{1}, x_{1+\frac{n}{3}}\right)$.

Let us consider now the mixed cycles generated by the coexistence of two cycles of $\phi$. 
Proposition 4. Let $X=\left\{x_{1}, x_{2}, \ldots, x_{n}\right\}$ be a cycle of $\phi(x)$ of period $n \geq 1$, and $Y=\left\{y_{1}, y_{2}, \ldots, y_{m}\right\}$ be a cycle of $\phi(x)$ of period $m \geq 1$, and let $L_{2}$ be the least common multiple between $n$ and $m$, then, besides the homogeneous cycles,

- $M$ has $(n+m) \frac{n m}{L_{2}}$ cycles of period $3 L_{2}$, if neither $n$ nor $m$ are multiples of 3 ;

- $M$ has $3(n+m) \frac{n m}{L_{2}}$ cycles of period $L_{2}$, if at least one between $n$ and $m$ is a multiple of 3 .

Proof. Once more, the tools for the proof are the claims of Sec.2.

If neither $n$ nor $m$ are multiples of 3 , also $\phi^{3}(x)$ has two different cycles of period $n$ and $m$. Then the map $M$ has $(n+m) \frac{n m}{L_{2}}$ mixed cycles of period $3 L_{2}$.

If $n$ (or $m$ ) is a multiple of $3, \phi^{3}(x)$ has three different cycles of period $\frac{n}{3}$ and 1 cycle of period $m$ and the least common multiple between $\frac{n}{3}$ and $m$ is $\frac{L_{2}}{3}$. Thus the mixed cycles are of period $L_{2}$ and are $\frac{(m+n)^{3}-n^{3}-m^{3}}{L_{2}}=$ $3(m+n) \frac{m n}{L_{2}}$ in number.

If both $n$ and $m$ are multiples of $3, \phi^{3}(x)$ has three different cycles of period $\frac{n}{3}$ and three different cycles of period $\frac{m}{3}$ and the least common multiple between $\frac{n}{3}$ and $\frac{m}{3}$ is $\frac{L_{2}}{3}$. Thus the mixed cycles are of period $L_{2}$ and are $\frac{(m+n)^{3}-n^{3}-m^{3}}{L_{2}}=3(m+n) \frac{m n}{L_{2}}$ in number.

Finally, we consider the pure mixed cycles generated by three coexisting cycles of $\phi$.

Proposition 5. Let $X=\left\{x_{1}, x_{2}, \ldots, x_{n}\right\}, Y=\left\{y_{1}, y_{2}, \ldots, y_{m}\right\}, Z=\left\{z_{1}, z_{2}, \ldots, z_{p}\right\}$ be three coexisting cycles of $\phi(x)$ of period $n, m$ and $p$, respectively. Then, besides the homogeneous cycles and the mixed ones, $M$ has

- $2 \frac{n m p}{L_{3}}$ cycles of period $3 L_{3}$, if $L_{3}$ is not a multiple of 3 ;

- $6 \frac{n m p}{L_{3}}$ cycles of period $L_{3}$, if $L_{3}$ is a multiple of 3

where $L_{3}=L C M\{n, m, p\}$.

Proof. If $L_{3}$ is not a multiple of 3 then $\phi^{3}$ has three coexistent cycles of period $n, m$ and $p$, respectively. Hence from the claim on pure mixed cycles of $T$ in (2) we immediately obtain that $M$ has $2 \frac{n m p}{L_{3}}$ pure mixed cycles of period $3 L_{3}$.

If $L_{3}$ is a multiple of 3 then at least one of the periods is a multiple of 3 as well. In the case where only one of the periods is a multiple of 3 , say $n$ to fix the ideas, then $\phi^{3}$ has a cycle of period $m$, a cycle of period $p$ and 3 cycles of period $\frac{n}{3}$. Each one of these latter cycles combined with the former ones gives rise to $2 \frac{\frac{n}{3} m p}{\frac{L_{3}}{3}}=2 \frac{n m p}{L_{3}}$ cycles of period $L_{3}$, since $L C M\left(\frac{n}{3}, m, p\right)=\frac{L_{3}}{3}$. Then we obtain that $M$ has $6 \frac{n m p}{L_{3}}$ coexisting cycles of period $L_{3}$.

If two periods are multiples of 3 , say $n$ and $m$, then $\phi^{3}$ has one cycles of period $p, 3$ cycles of period $\frac{n}{3}$ and 3 cycles of period $\frac{m}{3}$. Since there are 9 different ways to combine the coexistent cycles in groups of three and $\operatorname{LCM}\left(\frac{n}{3}, \frac{m}{3}, p\right)=\frac{L_{3}}{3}$. Therefore we obtain that the coexisting cycles of $M$ are $9 \cdot 2 \frac{\frac{n}{3} \frac{m}{3} p}{\frac{L_{3}}{3}}=6 \frac{n m p}{L_{3}}$ in number, all of period $L_{3}$. Analogously we obtain the assert when all the periods are multiple of 3 , since there are 27 different ways to combine the coexisting cycles of $\phi^{3}$ and $\operatorname{LCM}\left(\frac{n}{3}, \frac{m}{3}, \frac{p}{3}\right)=\frac{L_{3}}{3}$.

It is clear that if the map $\phi$ has more than three coexistent cycles, all the cycles of $M$ can be obtained by firstly considering all the homogeneous ones, then the mixed cycles generated by all the possible pairs of cycles and finally the pure mixed cycles generated by all the possible triplets.

\subsection{Local stability of the cycles}

As recalled in Section 2, also the stability properties of the cycles of the maps $T$ having separate third iterate can be obtained by one of the components of $T^{3}$. Being the components of the third iterate of $M$ in (1) all equal to $\phi^{3}$, we obtain that if $n$ is not a multiple of 3 and $\lambda$ is the multiplier of a $n$-cycle of $\phi$ then $\lambda^{3}$ is the multiplier of the corresponding cycle of $\phi^{3}$, while if $n$ is a multiple of 3 the three cycles of period $n / 3$ have as multiplier $\lambda$. Therefore we can apply the results related to map $T$ in (2) to the particular case we are analyzing.

Starting from the homogeneous cycles obtained in Proposition 3, we get that if $C$ is a period $n$ cycle of $\phi$ with multiplier $\lambda$ then 
- if $n$ is not a multiple of 3 , the cycle of period $n$ has eigenvalues $\left(\lambda, \lambda \frac{i \sqrt{3}-1}{2},-\lambda \frac{i \sqrt{3}+1}{2}\right)$ while the cycles of period $3 n$ have all the eigenvalues equal to $\lambda^{3}$;

- if $n$ is a multiple of 9 , all the cycles of period $n$ have eigenvalues $\lambda_{1}=\lambda_{2}=\lambda_{3}=\lambda$;

- if $n$ is a multiple of 3 but $\frac{n}{3}$ is not, the 3 cycles of period $\frac{n}{3}$ have eigenvalues $\left(\sqrt[3]{\lambda}, \sqrt[3]{\lambda} \frac{i \sqrt{3}-1}{2},-\sqrt[3]{\lambda} \frac{i \sqrt{3}+1}{2}\right)$ while the cycles of period $n$ have all the eigenvalues equal to $\lambda$.

Regarding the mixed and pure mixed cycles, we have that when the map $\phi$ has three coexisting cycles $A=$ $\left\{x_{i}\right\}_{i=1, \ldots n}, B=\left\{y_{i}\right\}_{i=1, \ldots m}$ and $C=\left\{z_{i}\right\}_{i=1, \ldots p}$ with multipliers $\lambda_{i}, i=A, B, C$ respectively, then the pure mixed cycles ${ }^{1}$ of period $3 L_{3}=3 L C M(n, m, p)$ have eigenvalues $\left(\lambda_{A}^{3 k_{1}}, \lambda_{B}^{3 k_{2}}, \lambda_{C}^{3 k_{3}}\right)$, where $k_{1}=L_{3} / n, k_{2}=L_{3} / m$, $k_{3}=L_{3} / p$ while those of period $L_{3}$ have eigenvalues $\left(\lambda_{A}^{k_{1}}, \lambda_{B}^{k_{2}}, \lambda_{C}^{k_{3}}\right)$.

Analogously, the eigenvalues of the mixed cycles, obtained for example by the combination of the periodic points of $A$ and $B$ depend on the components they have and may be given either by $\left(\lambda_{A}^{3 k_{1}}, \lambda_{A}^{3 k_{1}}, \lambda_{B}^{3 k_{2}}\right)$ or by $\left(\lambda_{A}^{3 k_{1}}, \lambda_{B}^{3 k_{2}}, \lambda_{B}^{3 k_{2}}\right)$ if they are of period $3 L_{2}=3 L C M(m, n)$ while if they are of period $L_{2}$ the eigenvalues may be either $\left(\lambda_{A}^{k_{1}}, \lambda_{A}^{k_{1}}, \lambda_{B}^{k_{2}}\right)$ or $\left(\lambda_{A}^{k_{1}}, \lambda_{B}^{k_{2}}, \lambda_{B}^{k_{2}}\right)$.

To summarize the results given above we state the following

Proposition 6. Any cycle $C$ of the three dimensional map $M$ is associated with one, or two or three cycles of $\phi$, say $C_{1}, C_{2}$ and $C_{3}$ eventually coincident, and

- (i) the periodic points of $C$ belong to the set $\mathbf{P}=\left(C_{1} \cup C_{2} \cup C_{3}\right)^{3}$;

- (ii) $C$ is attracting for $M$ iff $C_{1}, C_{2}$ and $C_{3}$ are attracting for $\phi$.

It is worth to remark that any bifurcation of a cycle of $\phi$ corresponds to a bifurcation of the map $M$ associated with 3 eigenvalues crossing simultaneously the unit circle.

\section{An example}

In this section, we present an example in order to illustrate the local and global properties of the map $M$ when the endomorphism $\phi$ is given. In particular we consider the quadratic unimodal map

$$
\phi(x)=x^{2}-b
$$

also known as Myrberg map. The dynamical behavior of (4) when the parameter $b$ is varied is well known (see, among others, [3]). At $b_{0}=-1 / 4$ a fold bifurcation occurs and when $b>b_{0}$ two fixed points appear

$$
p=\frac{1}{2}-\sqrt{\frac{1}{4}+b} \text { and } q=\frac{1}{2}+\sqrt{\frac{1}{4}+b}
$$

with multipliers $\lambda_{p}=1-\sqrt{1+4 b}$ and $\lambda_{q}=1+\sqrt{1+4 b}$, respectively. Then $q$ is always repelling, while $p$ is attracting until $b<b_{1}=3 / 4$. At $b=b_{1}$ a supercritical flip bifurcation occurs, that makes repelling the fixed point $p$ and gives rise to the appearance of an attracting cycle of period 2. Such a flip bifurcation is the first one of the well known cascade of period doubling bifurcations leading to chaotic dynamics.

In the following we shall study the effect of the coexistence of fixed points and period 2 cycles of the map $\phi$ looking in particular at the attractors of $M$ and at their basins of attraction.

\footnotetext{
${ }^{1}$ As they have been classified in Proposition 5
} 


\subsection{Coexistence of two fixed points}

Let us consider, as a first example, the Myrberg map with $b=0.1$. At this parameter value the map $\phi$ in (4) has two coexistent fixed points $p$, stable, and $q$ unstable. Due to the noninvertibility of the map, $q$ has two preimages of rank-1, $q$ itself and $r=-\left(\frac{1}{2}+\sqrt{\frac{1}{4}+b}\right)$. The interval $(r, q)$ is the basin of attraction of $p$ while $\mathbb{R} \backslash[r, q]$ is the set of the divergent trajectories.

The coexistence of two fixed points of $\phi$ implies that the map $M$ in (1) has 2 fixed points, $P$ and $Q$ (homogeneous cycles) and two period 3 cycles, $S$ and $N$, of mixed type (see Prop.3 and Prop.4). The fixed point $P=(p, p, p)$ is stable while $Q=(q, q, q)$ is unstable; both $S$ and $N$ are saddle cycles, having as eigenvalues $\left(\lambda_{p}, \lambda_{p}, \lambda_{q}\right)$ and $\left(\lambda_{p}, \lambda_{q}, \lambda_{q}\right)$, respectively. A qualitative draft of the mutual position of the coexistent cycles of $M$ is given in Fig.1a, where also the point $Q_{-1}=(r, r, r)$, a first rank preimage of $Q$, is represented.

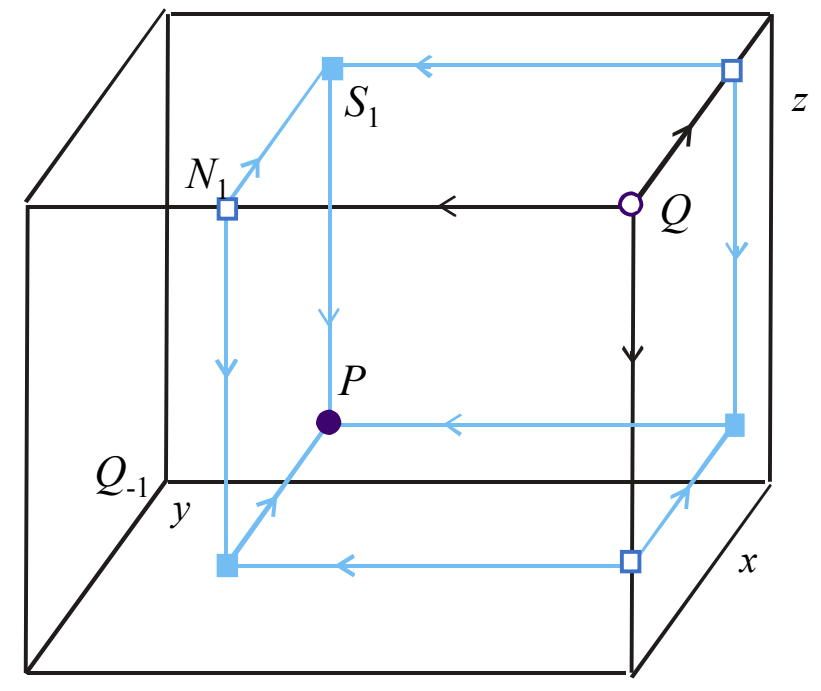

(a)

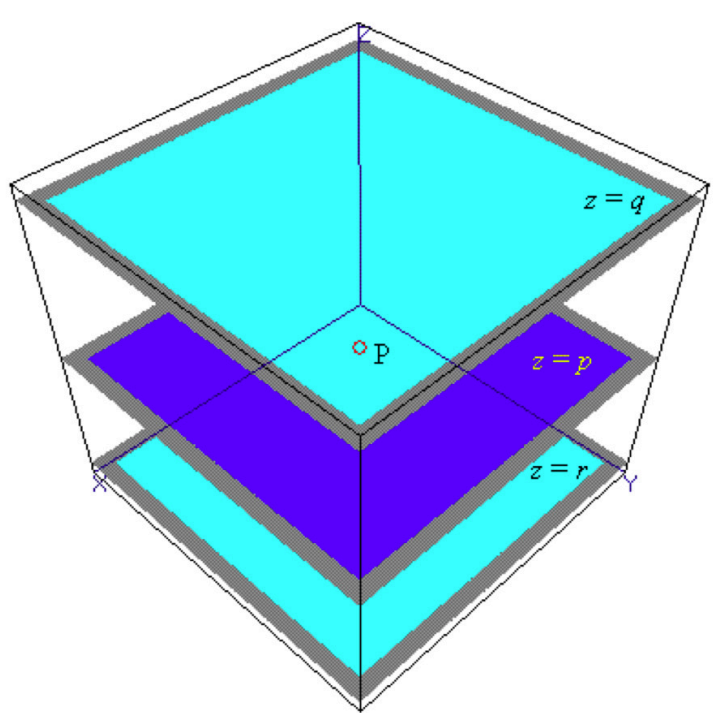

(b)

FIGURE 1. (a) The box B containing the two fixed points of $M$ and their coexisting saddle cycles. $S_{1}=(p, p, q)$ and $N_{1}=(q, p, q)$ are two periodic points of the saddle cycles $S$ and $N$, respectively (b) The three sections of the phase plane $z=r, z=p$ and $z=q$.

The unstable fixed point $Q$ has eight different rank-1 preimages, one of them being $Q$ itself; they are the vertex of a box $\mathbf{B}$ that contains the bounded trajectories. Let us consider the periodic point $S_{1}=(p, p, q)$ belonging to the saddle cycle $S$. Since the Jacobian matrix of $M^{3}$ evaluated in such a point is diagonal, we obtain that the portion of the plane $z=q$ included in $\mathbf{B}$ belongs to the stable set of the saddle cycle $S$ and it is invariant, as well as its forward iterates belonging to $x=q$ and $y=q$. To obtain the whole basin of attraction $\mathfrak{B}(P)$ of the stable fixed point $P$ we have to look also at the preimages of such planes; we obtain that also portions of the planes $x=r, y=r$ and $z=r$ are boundaries of $\mathfrak{B}(P)$, as illustrated in Fig.1a. Hence we can conclude that the basin of attraction of $P$ is a rectangular parallelepiped whose lateral surface is given by the planes parallel to the coordinate planes and passing through the point $Q$ and $Q_{-1}$.

The numerical simulation of Fig.1b confirms such a deduction, as the section $z=r, z=p$ and $z=q$ of the basins $\mathfrak{B}(P)$ show. 


\subsection{Coexistence of two fixed points and a cycle of period 2}

To consider a more complex situation, we set $b=0.95$ in the Myrberg map (4). Hence the first flip bifurcation has already occurred and the two fixed points $p$ and $q$ of $\phi$, both repelling, coexist with an attracting cycle $\left\{c_{1}, c_{2}\right\}$ of period 2. Let us denote $\lambda_{c}$ the multiplier of the period 2 cycle.

As illustrated in Fig.2a, $q$ and its rank-1 preimage $r$ still separate bounded and unbounded trajectories, while the repelling fixed point $p$ and its preimages separate the basins of attraction of the two fixed points $c_{1}$ and $c_{2}$ of the map $\phi^{2}$, second iterate of $\phi$.

By applying Propositions 3,4 and 5 we obtain that the map $M$ has

- two homogeneous stable cycles: one of period $2, C$, and one of period $6, \bar{C}$;

- four repelling cycles: the two fixed points, $P$ and $Q$ (homogeneous) and two mixed cycles of period 3;

- eight saddles cycles of period 6 , of mixed and pure mixed type.

Before to explaining the shape of the basins of attraction of $C$ and $\bar{C}$, let us remark that the occurrence of the flip bifurcation of the fixed point $p$ of the map $\phi$ has considerable consequences on the invariant sets of the map $M$, since it causes the sudden appearance of 14 cycles from the 4 previously existing.

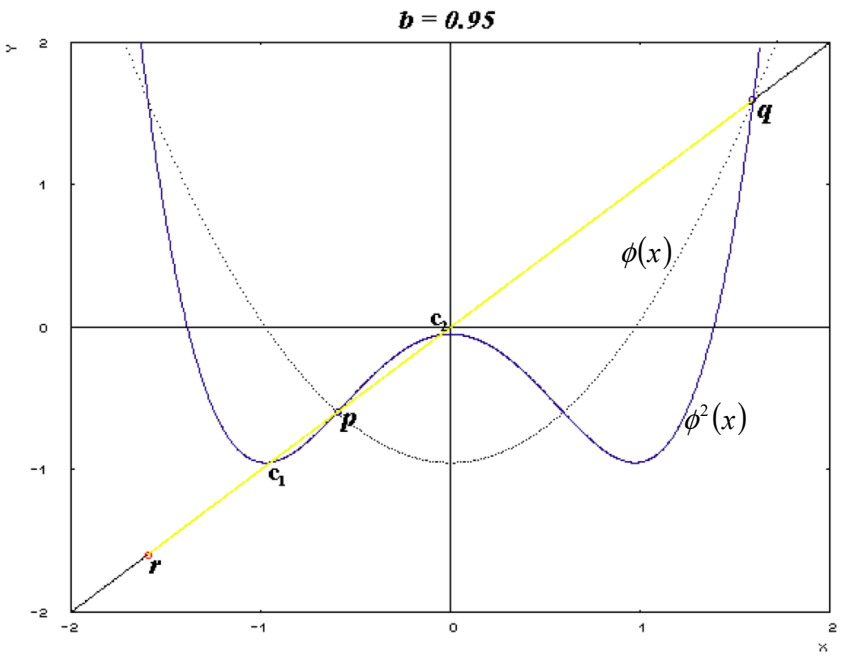

(a)

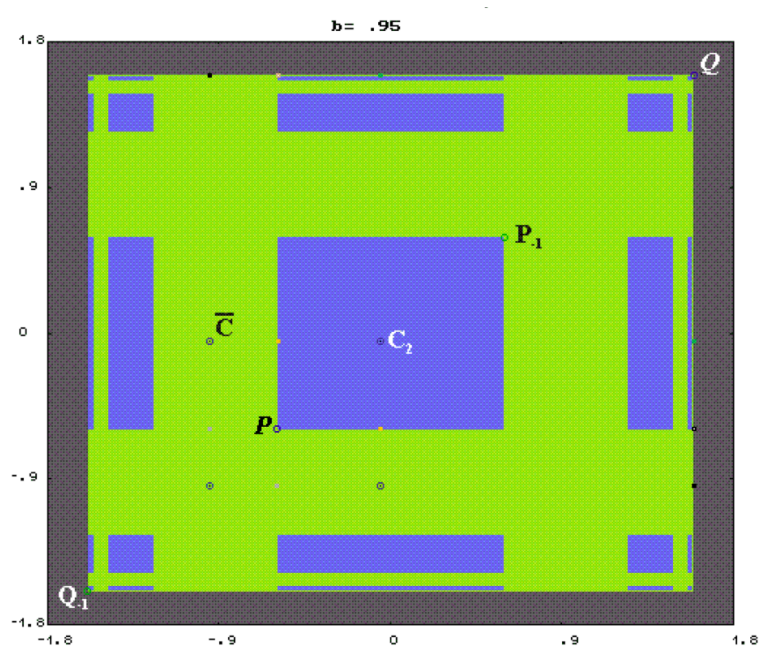

(b)

FiguRE 2. (a) The Myrberg map with $b=0.95$. (b) The section of the phase plane on the plane $z=c_{2}$.

From the section of the phase plane shown in Fig.2b, we can infer that the unstable fixed point $Q$ and the mixed cycles having at least a component equal to $q$ are involved in the boundary separating bounded and unbounded trajectories in a way similar to the one described in the previous subsection. Hence, we now focus our attention only on the cycles whose periodic points belong to the set $\left\{p, c_{1}, c_{2}\right\}^{3}$, that is on the fixed point $P=(p, p, p)$, the two attracting cycles $C$ and $\bar{C}$ (of period 2 and 6 , respectively) and the 3 saddle cycles of period $6, S$ and $\bar{S}$, having eigenvalues equal to $\left(\lambda_{c}, \lambda_{c}, \lambda_{p}\right)$, and $N$ with eigenvalues $\left(\lambda_{c}, \lambda_{p}, \lambda_{p}\right)$. They are qualitatively represented in Fig.3a.

To obtain the boundary separating the basins of attraction $\mathfrak{B}(C)$ and $\mathfrak{B}(\bar{C})$ we have to consider the three saddle cycles and their stable sets. Let us consider as an example the periodic point $S_{1}=\left(c_{2}, c_{2}, p\right)$ of the 


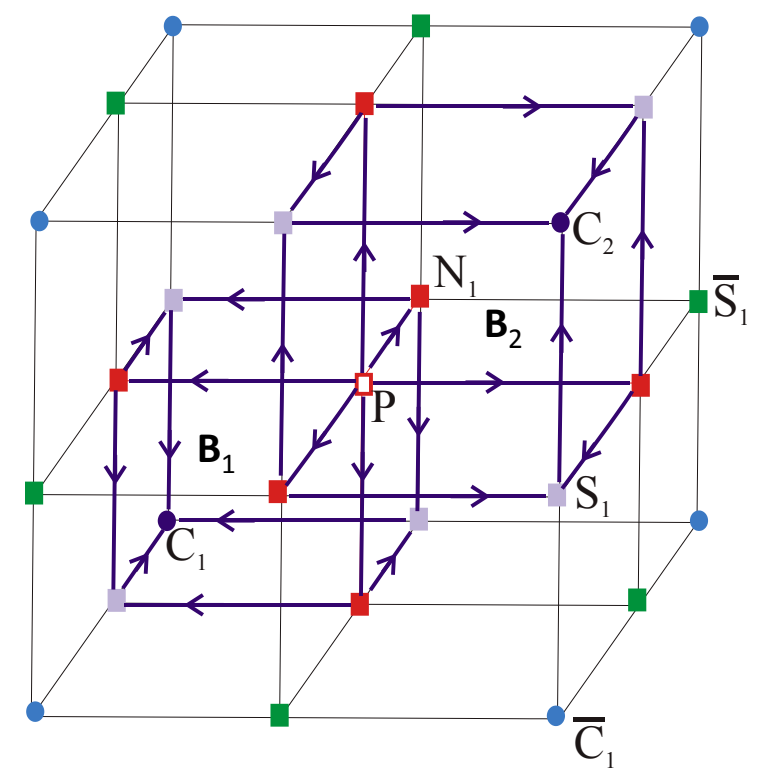

(a)

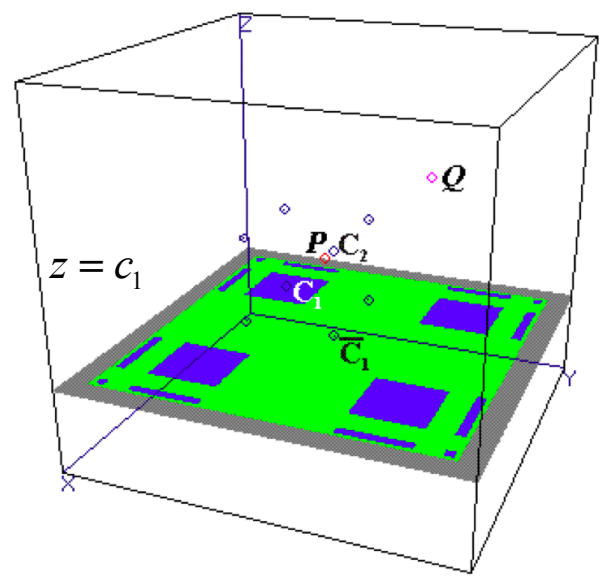

(b)

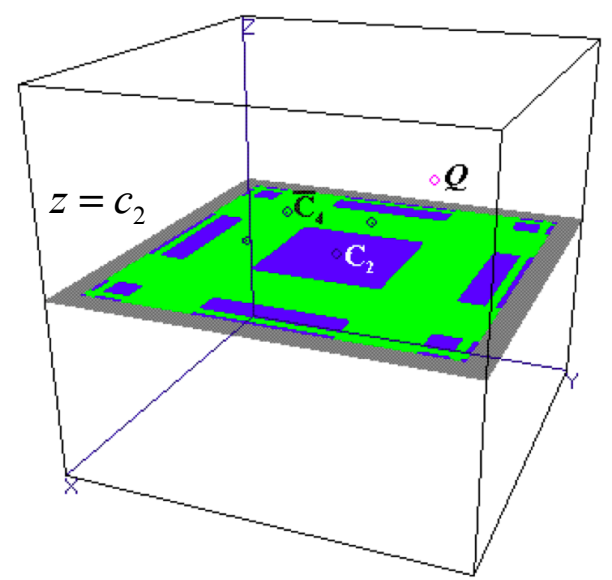

(c)

FiguRE 3. (a) The coexisting cycles of $M$ whose periodic points belong to the set $\left\{p, c_{1}, c_{2}\right\}^{3}$. The two boxes $\mathbf{B}_{1}$ and $\mathbf{B}_{2}$ are drawn in bold. (b) The section $z=c_{1}$ of the phase plane. (c) The section $z=c_{2}$ of the phase plane.

saddle $S$. Due to the existence of the saddle $N$, only a portion of the plane $z=p$ can be immediately identified as belonging to the stable set of $S$, that is, the one such that $x>p \wedge y>p$. Starting from such a portion, we build the whole stable set considering first its forward iterates and then their preimages of any rank. So doing, we obtain that the immediate basin ${ }^{2}$ of the cycle $C$ of period 2 is given by the union of two rectangular parallelepipeds, $\mathbf{B}_{1}$ and $\mathbf{B}_{2}$, bounded by the planes $z=p, y=p$ and $x=p$ and their rank-1 preimages and such that the points belonging to $\mathbf{B}_{2}$ have all the coordinates larger than $p$, while those belonging to $\mathbf{B}_{1}$ have all the coordinates smaller than $p$.

We also remark that the basin $\mathfrak{B}(\bar{C})$ is a multiply connected set (i.e., connected with holes) while the basin $\mathfrak{B}(C)$ is a disconnected set.

Once more the two sections of the phase plane shown in Fig.3b and Fig.3c and obtained by numerical simulation confirm our deductions.

It is now simple to understand that as the parameter $b$ is further increased the number of the coexisting cycles of the map $M$ quickly grows whenever a flip bifurcation of a cycle of $\phi$ occurs. Also the basins of attraction of the coexisting attractors become more and more complex, due to the noticeable number of saddles cycles

\footnotetext{
${ }^{2}$ That is, the two largest connected components of the basin, each one containing a periodic point
} 
appearing in the phase space, but they will be always given by the union of rectangular parallelepipeds having the faces parallel to the coordinates planes.

\section{REFERENCES}

[1] A. Agliari, D. Fournier-Prunaret and A.-K. Taha (2010). Periodic orbits and their bifurcations in 3-D maps having separate third iterate. In: Bischi G.I., Chiarella C., Sushko I. (Eds.) Global Analysis of Dynamic Models in Economics and Finance. BERLIN: Springer-Verlag, forthcoming.

[2] G.I. Bischi, C. Mammana and L. Gardini. Multistability and cyclic attractors in duopoly games. Chaos, Solitons E Fractals 11 (2000), 543-564.

[3] Mira, C., Gardini, L., Barugola, A., Cathala, J.C., Chaotic Dynamics in Two-Dimensional Noninvertible Maps, World Scientific, Singapore, 1998.

[4] F. Tramontana, L. Gardini, T. Puu, Cournot duopoly when the competitors operate multiple production plants. Journal of Economic Dynamics \& Control 33 (2009), 250-265. 